\title{
Aberrant expression of lysophosphatidic acid (LPA) receptors in human colorectal cancer
}

\author{
Dai Shida ${ }^{1}$, Toshiaki Watanabe ${ }^{1}$, Junken Aoki $^{2}$, Kotaro Hama ${ }^{2}$, Joji Kitayama ${ }^{1}$, \\ Hirofumi Sonoda ${ }^{2}$, Yasuhiro Kishi ${ }^{2}$, Hironori Yamaguchi ${ }^{1}$, Shin Sasaki ${ }^{1}$, Akihiro Sako ${ }^{1}$, \\ Tsuyoshi Konishi ${ }^{1}$, Hiroyuki Arai $^{2}$ and Hirokazu Nagawa ${ }^{1}$ \\ ${ }^{1}$ Department of Surgical Oncology, University of Tokyo Graduate School of Medicine, Tokyo, Japan and \\ ${ }^{2}$ Graduate School of Pharmaceutical Sciences, The University of Tokyo, Tokyo, Japan
}

\begin{abstract}
Lysophosphatidic acid (LPA) is a simple bioactive phospholipid with diverse effects on various cells, that interacts with three G protein-coupled transmembrane receptors, LPA1, LPA2, and LPA3. The expression pattern and functions of these LPA receptors in various tumors have not been fully examined, except in ovarian cancer. To evaluate the LPA receptor expression profile in human colorectal cancer and in normal mucosa, we used real-time reverse transcription-polymerase chain reaction (RT-PCR) and measured the expression levels of LPA1, LPA2, and LPA3 messenger RNA (mRNA) in 26 colorectal cancers and 16 corresponding normal tissue samples. Normal epithelium expressed both LPA1 and LPA2 mRNA at similar levels. In comparison, colorectal cancers expressed LPA1 mRNA at a significantly lower level (0.3-fold; $P<0.05)$, and LPA2 mRNA at a significantly higher level (three-fold; $\boldsymbol{P}<\mathbf{0 . 0 5}$ ), as compared with normal tissues. Thus, the ratio of LPA2/LPA1 increased markedly during malignant transformation (18-fold increase). LPA3 mRNA was expressed at only a low level in both normal and cancer tissues. We also assessed LPA2 expression immunohistochemically using a rat anti-LPA2 monoclonal antibody, and confirmed high expression of LPA2 in colorectal cancer at the protein level. As for LPA1, we examined Western blot analysis for $\mathbf{1 6}$ matched normal and cancer tissues. It revealed a significant decrease in the expression of LPA1 protein in cancer tissues compared to normal mucosa in nine of 16 cases, and in the remaining seven cases the expression levels was much the same. These results suggested that alteration of LPA receptor expression might be an important event in the development of colorectal cancer, and therefore, LPA and its receptors could be a chemopreventive target against colorectal cancer.

Laboratory Investigation (2004) 84, 1352-1362, advance online publication, 28 June 2004; doi:10.1038/labinvest.3700146
\end{abstract}

Keywords: aberrant expression of LPA2; carcinogenesis; colorectal cancer; LPA receptor; lysophosphatidic acid; phospholipid

Lysophosphatidic acid (LPA, 1- or 2-acyl-sn-glycero3-phosphate), the simplest glycerophospholipid, mediates a broad range of cellular responses, including smooth muscle cell contraction, platelet aggregation, neurite retraction/cell rounding, regulation of cell proliferation, protection from apoptosis, modulation of chemotaxis, and transcellular migration. ${ }^{1-3}$ Some of these cellular responses implicate LPA as a mediator of tumor progression. ${ }^{4}$ LPA is present at high levels in ascitic fluid from ovarian cancer patients ${ }^{5-7}$ and is known to be an 'ovarian cancer activating factor'. ${ }^{8}$ Previous reports have demonstrated that LPA stimulates prolifera-

Correspondence: Dr D Shida, MD, Department of Surgical Oncology, University of Tokyo Graduate School of Medicine, 7-3-1 Hongo, Bunkyo-ku, Tokyo 113-8655, Japan.

E-mail: SHIDA-DIS@h.u-tokyo.ac.jp

Received 6 January 2004; revised 15 May 2004; accepted 24 May 2004; published online 28 June 2004 tion, migration, matrix metalloproteinase (MMP) activation, and secretion of angiogenic factors in ovarian cancer cells, suggesting a positive role in the development of ovarian cancer. However, the relevance of LPA to other types of human malignancy is not well known. ${ }^{6,9-13}$

LPA has been shown to interact with at least three receptors, LPA1/Edg-2, LPA2/Edg-4, and LPA3/ Edg-7. ${ }^{14,15}$ These LPA receptors differ with respect to their distribution in various normal tissues. LPA1 is broadly expressed in various normal tissues, whereas expression of LPA2 and LPA3 is more restricted, which may account for the various biological effects of LPA. ${ }^{14,15}$ Using gene-targeting methods, Contos et $a l^{16,17}$ showed that LPA1 and LPA2 had redundant functions in mediating multiple endogenous LPA responses including phospholipase C activation, calcium mobilization, cell proliferation, and stress fiber formation in mouse embryonic fibroblasts. However, LPA1 and LPA2 
knockout mice showed quite different phenotypes. Deletion of lpa1 in mice results in craniofacial dysmorphism, semilethality due to defective suckling behavior, and generation of a small fraction of pups with frontal hematoma. ${ }^{16}$ In contrast, lpa2(-/-) mice were born at the expected frequency and displayed no obvious phenotypic abnormalities. ${ }^{17}$ These results clearly indicate the different roles of LPA1 and LPA2 in vivo. It has been recently shown that, in ovarian and thyroid cancers, malignant transformation resulted in aberrant expression of LPA2 (and LPA3 in ovarian cancer), suggesting that shifts of LPA receptor expression during malignant transformation were involved in ovarian and thyroid carcinogenesis. ${ }^{18-21}$ Thus, LPA and its receptors seem to have a potential role in tumor biology, but the expression patterns of these LPA receptors in other tumors have not been satisfactorily examined.

Using various human colon cancer cells, we previously reported that LPA stimulated migration, adhesion, proliferation, and secretion of angiogenic factors in vitro, although LPA induced some different responses to those cells, which seemed to be based on their expression pattern of LPA receptors. ${ }^{22}$ Colorectal cancers are often associated with local bleeding and thus, at the tumor site, platelets are activated and secrete lysophospholipids such as lysophosphatidylcholine (LPC), lysophosphatidylethanolamine (LPE), and lysophosphatidylserine (LPS). These lysophospholipids are subsequently converted to LPA by lysophospholipase D. ${ }^{23}$ Therefore, significant amounts of extracellular LPA are thought to be present in colorectal cancer tissue, and able to induce various important biological responses in the cancer. In this study, we examined the expression profile of LPA receptors in human colorectal cancer tissue as well as normal tissue at both the messenger RNA (mRNA) level and protein level, in order to better understand the potential role of LPA in the development of colorectal cancer.

\section{Materials and methods}

\section{Reagents and Cell Cultures}

1-Oleoyl-LPA was purchased from Sigma Chemical Co. (St Louis, MO, USA). Recombinant human epidermal growth factor (EGF) was purchased from PeproTechec Ltd (London, UK). The human colon cancer cell line COLO320, WiDr, LoVo and HT29 were maintained in Dulbecco's modified Eagle medium (DMEM), and HCT116 were maintained in McCoy's 5a medium (Gibco BRL Co., Grand Island, NY, USA), supplemented with $10 \%$ fetal calf serum (Sigma), $100 \mathrm{U} / \mathrm{ml}$ penicillin, and $100 \mu \mathrm{g} / \mathrm{ml}$ streptomycin (Gibco). Rabbit polyclonal antihuman LPA1 antibody was purchased from Exalpha Biologicals, Inc. (Boston, MA, USA). Mouse monoclonal antihuman phosphotyrosine antibody, rabbit polyclonal anti-human epidermal growth factor receptor
(EGFR) antibody and rabbit polyclonal anti-human actin antibody were purchased from Santa Cruz Biotechnology (Santa Cruz, CA, USA). Dioctylglycerol pyrophosphate (DGPP 8:0), recently identified as antagonists of LPA1 and LPA3 receptors but not of the LPA2 receptor, ${ }^{24}$ was obtained from Avanti Polar Lipids, Inc. (Alabaster, AL, USA).

\section{Patients}

A total of 26 patients with colorectal cancer who underwent surgical resection in the Department of Surgical Oncology of the University of Tokyo between July 2001 and January 2003 were enrolled in this study. Right-sided tumors were classified as those originating proximal to the splenic flexure, and left-sided tumors as those located distal to or at the splenic flexure, including rectal cancers. Histological examination was performed routinely for all tumors, and all were diagnosed as adenocarcinoma. Histological findings were classified according to the UICC classification (G1, well differentiated; G2, moderately differentiated; G3, poorly differentiated). All cases were staged according to the American Joint Commission on Cancer (AJCC) 'TNM' system. This study was carried out with the approval of the Local Ethics Committee of the University of Tokyo, and written informed consent was obtained from all patients who participated.

\section{Isolation of Total RNA and Reverse Transcription}

Tumor tissue from the resected primary lesion and paired nontumor tissue (taken $10 \mathrm{~cm}$ away from the neoplasm) were immediately frozen in liquid nitrogen and kept at $-80^{\circ} \mathrm{C}$ until the extraction of RNA. Total RNA was extracted from each sample by the acid guanidine isothiocyanate/phenol/chloroform extraction method as described by Chomczynski and Sacchi. ${ }^{25}$ Then, $1 \mu \mathrm{g}$ of total RNA was reversetranscribed using a SuperScript First-Strand Synthesis System (Invitrogen Co., Carlsbad, CA, USA). The reverse transcription (RT) reaction was carried out in a total volume of $20 \mu \mathrm{l}$ according to the manufacturer's instructions. The complementary DNA (cDNA) was stored at $-20^{\circ} \mathrm{C}$ until use.

\section{Preparation of cDNA Calibrators}

We used pcDNA3 vector (Invitrogen) containing LPA1, LPA2, or LPA3 as the standard cDNA for each LPA receptor, as described previously. ${ }^{15}$ cDNA for human $\beta$-actin was prepared using human colon cDNA as template DNA and the following oligonucleotides: ccatcgaattcaccaccatggatgatgatatcgccgcgctc, aaggtgcggccgcctagaag catttgcggtggacgat. The resulting DNA fragments were digested with EcoRI/NotI and ligated into pBlueScript (Strategene, La Jolla, CA, USA). DNA sequence of cDNA prepared by RT-PCR 
were confirmed by DNA sequencing and used as the calibrator of $\beta$-actin.

\section{Real-Time Fluorescence Quantitative PCR}

The primers used in the analysis of LPA1, LPA2, LPA3, and $\beta$-actin gene expression are given in Table 1. All the primers were designed using the Primer Express software (Applied Biosystems, Foster, CA, USA). Real-time PCR reactions were performed in an ABI PRISM 7000 (Perkin-Elmer/ Applied Biosystems, Foster, CA, USA) using SYBR Green I (Perkin-Elmer) with the following profile: one step at $50^{\circ} \mathrm{C}$ for $2 \mathrm{~min}$, one step at $95^{\circ} \mathrm{C}$ for $10 \mathrm{~min}$, and 40 cycles at $95^{\circ} \mathrm{C}$ for $30 \mathrm{~s}$ and $60^{\circ} \mathrm{C}$ for $1 \mathrm{~min}$. Thermocycling was performed in a final volume of $20 \mu \mathrm{l}$ containing $1 \mu \mathrm{l}$ of cDNA sample. The ABI Prism software constructed a calibration curve by plotting the crossing point (Cp) vs the logarithm of the number of copies for each calibrator. The numbers of copies in unknown samples were calculated by comparing their Cp's with the calibration curve. To correct for differences in both RNA quality and quantity between samples, data were normalized using the ratio of the target cDNA concentration to that of $\beta$-actin.

\section{Monoclonal Antibodies against Human LPA2}

A peptide consisting of the carboxy-terminal (C-terminal) 17 amino acids of human LPA2 (335-351) was conjugated with keyhole limpet hemocyanin. The conjugate was injected into the hind foot pads of WKY/Izm rats using Freund's complete adjuvant. The enlarged medial iliac lymph nodes from the rats were used for cell fusion with mouse myeloma cells, PAI. The antibody-secreting hybridoma cells were selected by screening with ELISA, immunofluorescence and Western blotting. Several clones were established. In this study, monoclonal antibody from clone 10D5 (rat IgG1) was used for immunohistochemical staining.

\section{Immunohistochemical Staining of LPA2}

The streptavidin-biotin peroxidase complex technique was used for staining sections. Formalin-fixed, paraffin-embedded sections (3- $\mu \mathrm{m}$ thick) of color- ectal cancer or normal tissue were dewaxed for 15 min in xylene and hydrated by passage through a graded ethanol series to tap water. Antigen retrieval was performed routinely by incubation in target retrieval solution (Dako, Tokyo, Japan) at $97^{\circ} \mathrm{C}$ for $20 \mathrm{~min}$. After blocking with $0.3 \%$ hydrogen peroxide in methanol, the sections were blocked for another 30 min with $10 \%$ normal rabbit serum. Rat antihuman LPA2 monoclonal antibody was then added. After incubation at $4^{\circ} \mathrm{C}$ for $18 \mathrm{~h}$, sections were incubated with a second biotinylated rabbit antibody with anti-mouse specificity (Nichirei, Tokyo, Japan) for $20 \mathrm{~min}$. Avidin-peroxidase reagent (Nichirei) was then applied for $10 \mathrm{~min}$. All sections were counterstained with hematoxylin. Reactivity was visualized with $3,3^{\prime}$-diaminobenzidine as the substrate, yielding a brown reaction product. The sections were then dehydrated through a graded ethanol series to xylene and mounted. All cases had a negative control that was run simultaneously with the test slide, in which the primary antibody was omitted. Evaluation of staining was performed in at least 10 random highpower fields $(\times 200)$ for each sample.

\section{Western Blot Analysis of LPA1}

Protein was extracted from 16 matched cancer and normal tissues, after total RNA was extracted from each sample. A measure of $20 \mu \mathrm{g}$ of protein was electrophoresed in sodium dodecyl sulfate (SDS)$15 \%$ polyacrylamide gel for $45 \mathrm{~min}$ at $200 \mathrm{~V}$. Then, the protein was transferred onto an Immobilon transfer membrane (Millipore Co., Bedford, MA, USA) for sequential incubation with $5 \%$ reconstituted nonfat milk powder to block unspecific sites, dilutions of rabbit polyclonal anti LPA1 antibody, and then horseradish peroxidase (HRP)-labeled donkey anti-rabbit IgG, prior to development with a standard ECL kit (Amersham, Inc., Buckinghamshire, England).

\section{Immunoprecipitation and Western Blot Analysis}

Colon cancer cells were starved in serum-free medium overnight and LPA or EGF was added to the culture. Some cells were pretreated with 0.1-10 $\mu \mathrm{M}$ DGPP for $30 \mathrm{~min}$. After incubation of starved cancer cells with $10 \mu \mathrm{M}$ LPA or $10 \mathrm{ng} / \mathrm{ml}$

Table 1 Primer sequences used for PCR analysis

\begin{tabular}{|c|c|c|c|}
\hline & Forward & Reverse & $\begin{array}{l}\text { Product } \\
\text { size (bp) }\end{array}$ \\
\hline$\beta$-Actin & $5^{\prime}$-atgaagatcaagatcattgctcctc-3' (nucleotides 1048-1072) & $5^{\prime}$-acatctgctggaaggtggaca-3' (nucleotides 1141-1121) & 94 \\
\hline LPA1 & $5^{\prime}$-aatcgggataccatgatgagtctt- $3^{\prime}$ (nucleotides 1138-1161) & $5^{\prime}$-ccaggagtccagcagatgataaa-3' (nucleotides 1214-1192) & 77 \\
\hline LPA2 & $5^{\prime}$-cgctcagcctggtcaagact- $3^{\prime}$ (nucleotides $713-732$ ) & $5^{\prime}$-ttgcaggactcacagcctaaac-3' (nucleotides $821-800$ ) & 109 \\
\hline LPA3 & 5'-aggacacccatgaagctaatgaa-3' (nucleotides 736-758) & $5^{\prime}$-gccgtcgaggagcagaac-3' (nucleotides $831-814$ ) & 96 \\
\hline
\end{tabular}

All of the primers were designed using the Primer Express software. 
EGF for 2 min, cellular protein lysates were obtained and then proteins were incubated with anti-EGFR antibody. Immunoprecipitates were collected with protein A-agarose. Immunoprecipitated proteins were electrophoresed in SDS-7.5\% polyacrylamide gel for $35 \mathrm{~min}$ at $200 \mathrm{~V}$. Then, the protein was transferred onto an Immobilon transfer membrane for sequential incubation with $5 \%$ reconstituted nonfat milk powder to block unspecific sites, dilutions of mouse monoclonal antiphosphotyrosine antibody, and then HRP-labeled donkey anti-rabbit IgG, prior to development with a standard ECL kit.

\section{Statistical Analysis of Data}

The results were statistically tested by paired and unpaired Student's $t$-tests when appropriate, using commercially available software (StatView-J 5.0, SAS Institute Inc., Cary, NC, USA). Results are given as mean \pm s.e. Differences between normal and cancer tissues were considered significant at $P<0.05$.

\section{Results}

\section{Sensitivity and Specificity of PCR}

To test the sensitivity of the method based on $\mathrm{Cp}$ values, calibration curves were prepared for LPA1, LPA2, and LPA3 cDNAs from known quantities of cDNA (each diluted 10-fold from $10^{5}$ to $10^{2}$ copies of cDNA per reaction mixture). All calibration curves showed correlation coefficients $>0.99$, indicating a precise log-linear relation (Figure 1a). The detection limit using the specific primers shown in Table 1 was the same (10 copies of cDNA) for all three target genes. First, we examined the expression of LPA1, LPA2, and LPA3 mRNA in five human colon cancer cells, COLO320, LoVo, HT29, WiDR, and HCT116 cells. As shown in Figure 1b, COLO320 and HCT116 cells expressed considerable levels of LPA1. LoVo, and HT29, and WiDR cells expressed LPA2 exclusively among the three LPA receptors. A significant amount of LPA3 was expressed in COLO320 and HCT116 cells (Figure 1b). These results were fully consistent with our previous data determined by Northern blot analysis. ${ }^{22}$

\section{Patient Characteristics and Quantification of LPA Receptor mRNA in Colorectal Cancer Tissue and Normal Tissue}

Patient characteristics are summarized in Table 2. In total, 26 patients (15 male and 11 female) were enrolled. The median age was 63.2 years (range, 27-89 years). The expression of LPA1, LPA2, LPA3, and $\beta$-actin mRNA was evaluated in these 26 colorectal cancer tissue samples and 16 corresponding normal tissue samples. Figure 2 shows mRNA values of the three LPA receptors normalized to $\beta$ -
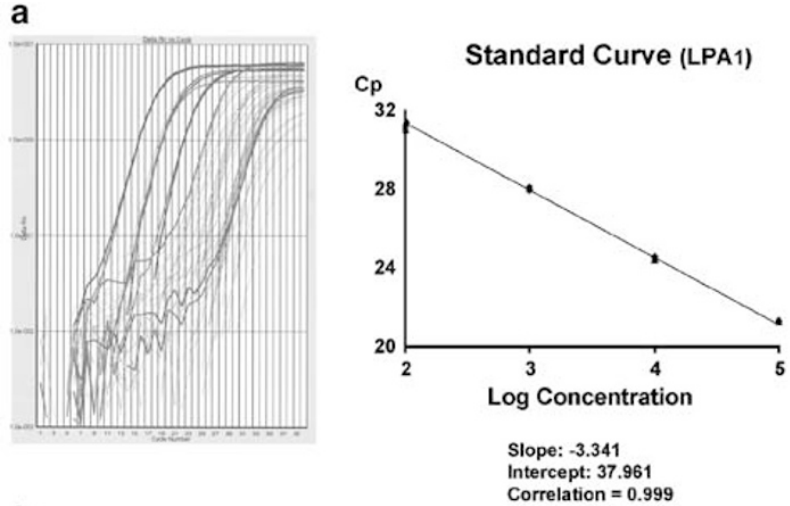

b

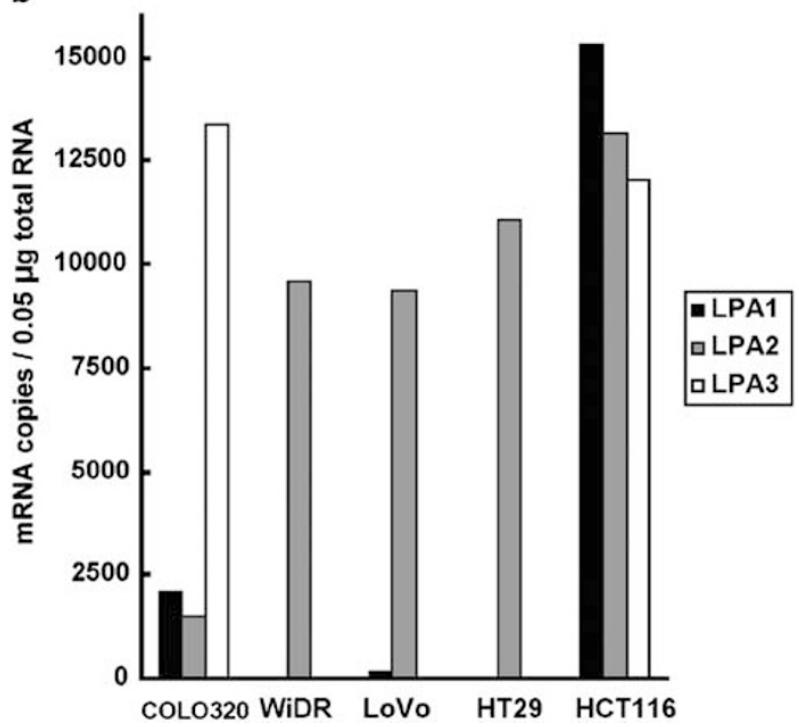

Figure 1 (a) Fluorescence data for LPA1 calibrators (right) together with the resulting calibration curve generated by the ABI Prism software (left). Solid lines indicate LPA1 cDNA plasmid diluted from $10^{5}$ to $10^{2}$ copy numbers assayed in triplicate. ABI Prism software creates a standard curve or the given concentration of each standard vs their Cp's. A linear relation was obtained between the threshold and logarithm of the starting copy number of LPA1. From this standard curve, one can determine the concentration of an unknown sample, expressed as an absolute value. Similar results were obtained when PCR products of LPA2 and LPA3 were analyzed. (b) Expression of LPA1, LPA2, and LPA3 mRNA of colon cancer cells, COLO320, WiDr, LoVo, HT29, and HCT116 cells, measured by real-time RT-PCR. RNA $(0.05 \mu \mathrm{g})$, extracted from colon cancer cells, was analyzed for mRNA of LPA receptors, using real-time RT-PCR.

actin mRNA in normal and cancer tissues. Each mRNA level was determined as the ratio to $\beta$-actin mRNA copies $\times 10^{8}$. Normal tissues expressed LPA1 mRNA as well as LPA2 mRNA in similar amounts (LPA1: $1143 \pm 196$, LPA2: $1110 \pm 245$ ). In comparison, colorectal cancer tissues expressed a significantly lower level of LPA1 mRNA (LPA1: $300 \pm 43$; $P<0.05)$ than normal tissues. In clear contrast, expression of LPA2 mRNA was markedly increased in cancer tissues (LPA2: $2400 \pm 294 ; P<0.05)$. When the levels of mRNA in 16 matched cancer and normal tissues were compared, the expression of LPA1 mRNA was decreased in all cases, whereas that of LPA2 was increased in 14 cases. The ratio of 
Table 2 Characteristics of patients

\begin{tabular}{|c|c|c|c|c|c|c|c|c|c|c|c|c|}
\hline Patient & Age (years) & Gender & site & Grade & $p T$ & Stage & $L P A 1(N)$ & $L P A 2(N)$ & $L P A 1(T)$ & $L P A 2(T)$ & $L P A 2 / L P A 1(N)$ & $L P A 2 / L P A 1(T)$ \\
\hline 1 & 64 & $\mathrm{~F}$ & S & G1 & T3 & II & 404 & 501 & 335 & 2480 & 1.24 & 7.4 \\
\hline 2 & 49 & M & $\mathrm{S}$ & G1 & T3 & IV & 439 & 382 & 125 & 844 & 0.87 & 6.7 \\
\hline 3 & 79 & M & $S$ & G1 & $\mathrm{T} 4$ & III & ND & ND & 96 & 866 & ND & 9 \\
\hline 4 & 49 & M & A & G1 & $\mathrm{T} 3$ & III & 1071 & 762 & 249 & 1098 & 0.71 & 4.4 \\
\hline 5 & 67 & $\mathrm{~F}$ & $\mathrm{~S}$ & G1 & T3 & IV & ND & ND & 435 & 1665 & ND & 3.8 \\
\hline 6 & 69 & $\mathrm{~F}$ & $\mathrm{R}$ & G1 & $\mathrm{T} 1$ & I & 922 & 369 & 297 & 3331 & 0.4 & 11.2 \\
\hline 7 & 38 & $\mathrm{M}$ & $\mathrm{R}$ & G1 & T3 & IV & 423 & 406 & 210 & 2642 & 0.96 & 12.6 \\
\hline 8 & 79 & $\mathrm{~F}$ & C & G1 & T3 & II & ND & ND & 166 & 855 & ND & 5.2 \\
\hline 9 & 71 & $\mathrm{~F}$ & A & G1 & T3 & IV & ND & ND & 174 & 1021 & ND & 5.8 \\
\hline 10 & 61 & M & $\mathrm{R}$ & G1 & T3 & II & ND & ND & 270 & 2383 & ND & 8.8 \\
\hline 11 & 77 & $\mathrm{~F}$ & A & G2 & T3 & II & ND & ND & 667 & 1899 & ND & 2.8 \\
\hline 12 & 65 & M & $\mathrm{R}$ & G1 & T1 & I & 600 & 286 & 475 & 1785 & 0.47 & 3.8 \\
\hline 13 & 85 & $\mathrm{~F}$ & $\mathrm{~T}$ & G3 & T3 & II & ND & ND & 210 & 1297 & ND & 6.2 \\
\hline 14 & 27 & $\mathrm{M}$ & $\mathrm{R}$ & G1 & $\mathrm{T} 4$ & II & 586 & 409 & 144 & 759 & 0.7 & 5.3 \\
\hline 15 & 53 & $\mathrm{~F}$ & $\mathrm{~S}$ & G1 & T3 & III & 576 & 1148 & 93 & 993 & 1.99 & 10.7 \\
\hline 16 & 59 & $\mathrm{~F}$ & S & G1 & T3 & III & 917 & 708 & 30 & 3365 & 0.77 & 112.2 \\
\hline 17 & 81 & M & $\mathrm{S}$ & G1 & T3 & II & 942 & 377 & 219 & 1664 & 0.4 & 7.6 \\
\hline 18 & 67 & $\mathrm{~F}$ & $\mathrm{R}$ & G1 & T3 & II & 2042 & 1564 & 381 & 3956 & 0.77 & 10.4 \\
\hline 19 & 89 & M & D & G1 & $\mathrm{T} 2$ & I & ND & ND & 48 & 6330 & ND & 131.9 \\
\hline 20 & 67 & M & $\mathrm{R}$ & G2 & T3 & IV & 1587 & 517 & 346 & 3576 & 0.33 & 10.3 \\
\hline 21 & 58 & M & $\mathrm{R}$ & G1 & T3 & IV & 1141 & 2392 & 299 & 2730 & 2.1 & 9.1 \\
\hline 22 & 54 & M & $\mathrm{R}$ & G1 & T3 & IV & 1027 & 2966 & 456 & 6122 & 2.89 & 13.4 \\
\hline 23 & 53 & M & C & G1 & $\mathrm{T} 2$ & I & ND & ND & 42 & 2695 & ND & 64.2 \\
\hline 24 & 54 & M & $\mathrm{R}$ & G2 & T3 & III & 2704 & 1775 & 410 & 1535 & 0.66 & 3.7 \\
\hline 25 & 67 & M & $\mathrm{D}$ & G1 & $\mathrm{T} 3$ & II & 2910 & 3198 & 652 & 3633 & 1.1 & 5.6 \\
\hline 26 & 61 & $\mathrm{~F}$ & A & G1 & T3 & IV & ND & ND & 973 & 2885 & ND & 3 \\
\hline
\end{tabular}

M, male; F, female; C, cecum; A, ascending colon; T, transverse colon; D, descending colon; S, sigmoid colon; R, rectum; grade, histological grade; pT, pathologic T stage (TNM classification by UICC staging); LPA1, LPA2, (target gene mRNA copies/ $\beta$-actin mRNA copies ) $\times 10^{8}$; N, normal mucosa; T, cancer; ND, not detected; LPA2/LPA1, ratio of LPA2/LPA1.
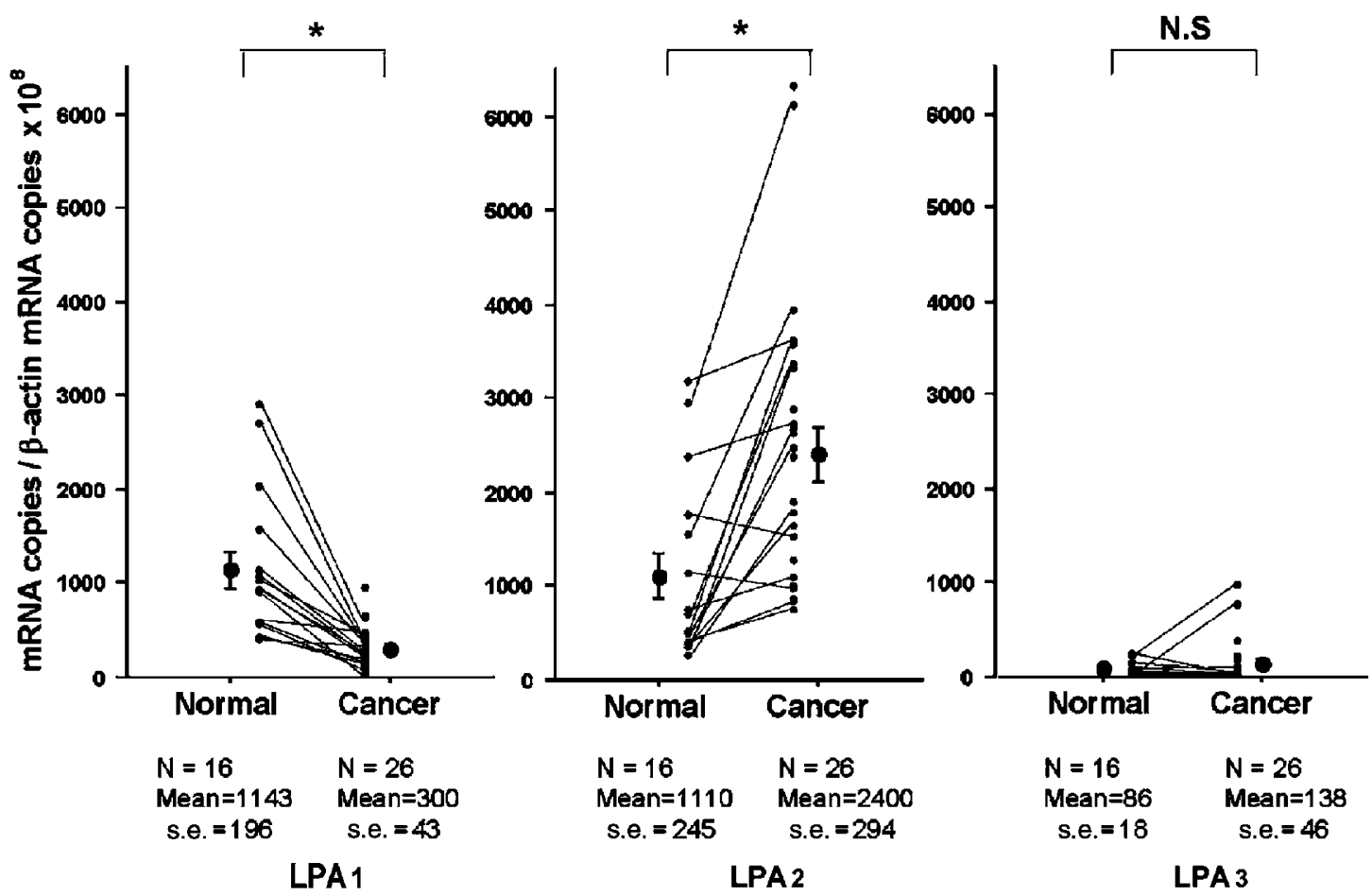

Figure 2 Expression of LPA1, LPA2, and LPA3 mRNA of colorectal tissues, measured by real-time RT-PCR. RNAs were extracted from cancer and normal tissues. They were analyzed for mRNAs of LPA receptors, using real-time RT-PCR. mRNA levels of the three LPA receptors were normalized to the $\beta$-actin mRNA level, and the level of each mRNA was estimated as the ratio compared with $\beta$-actin mRNA copies $\times 10^{8}$. (a) LPA1, (b) LPA2, and (c) LPA3. Mean values ( \pm s.e.) are also shown. ${ }^{*}$ indicates $P<0.05$ by Student's $t$-test. 


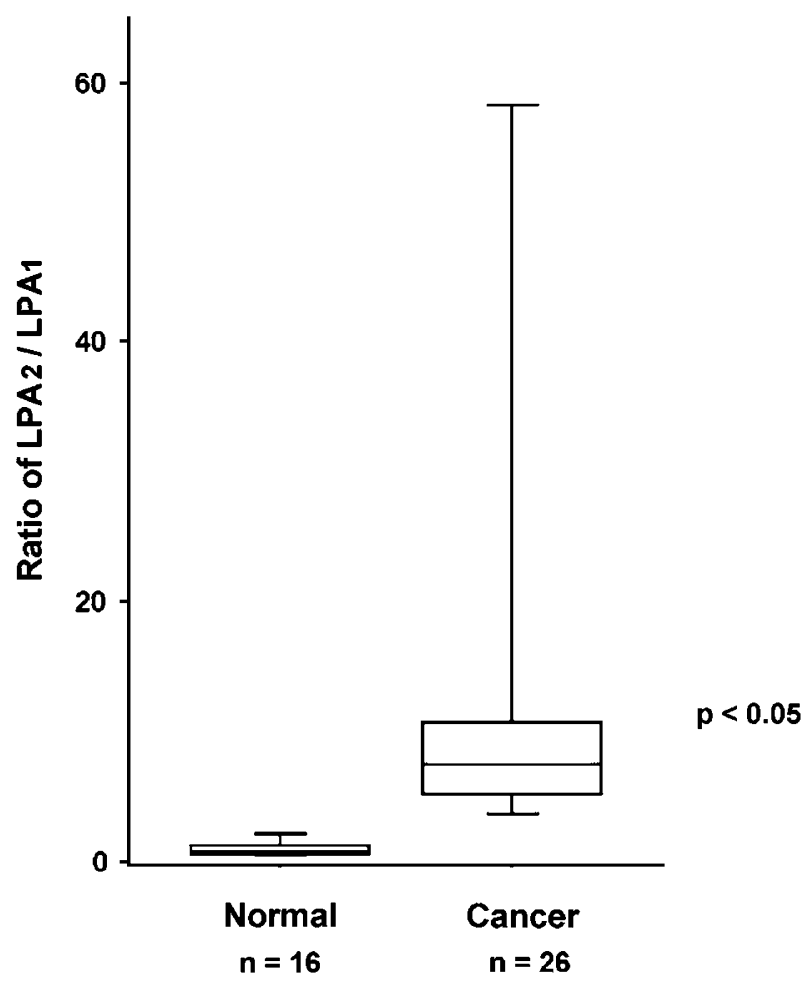

Figure 3 Ratio of LPA2/LPA1 mRNA in cancer and normal tissues. Data are shown as box chart plots with markings that indicate the 10th, 25th, 50th, 75th, and 90th percentiles. The difference in ratio between these two groups was statistically significant $(P<0.05$, by Student's $t$-test $)$.

LPA2/LPA1 in normal tissues was $1.0 \pm 0.2$, whereas that in cancer tissues was $18.0 \pm 6.3$ (Figure 3, Table 2). Thus, the ratio of LPA2/LPA1 was strikingly increased in cancer tissues $(P<0.05)$. On the other hand, the expression of LPA3 mRNA was low in both normal and cancer tissues, and with no significant difference between the two (Normal: $86 \pm 18$, Cancer: $138 \pm 46 ; P=0.39$ ). We examined the correlation between the expression of LPA receptors and various clinicopathological factors. However, neither LPA1 mRNA level nor LPA2 mRNA level nor the ratio of LPA2/LPA1 correlated with tumor depth, lymph node metastasis, histological grading, or TNM stage (Table 2). Comparing the Stage IV group with the others, the LPA1 level in the Stage IV group was higher than that in the others, but the difference was not statistically significant (Stage IV: $377 \pm 95$, others: $266 \pm 46 ; P=0.24$ ). As for tumor site, no significant difference was observed between right-sided (LPA1: $354 \pm 127$, LPA2: $1679 \pm 314$, ratio of LPA2/LPA1: $13.1 \pm 8.5$ ) and leftsided cancers (LPA1: $280 \pm 38$, LPA2: $2666 \pm 370$, ratio of LPA2/LPA1: $20.1 \pm 8.3)(P=0.46,0.14$, and 0.63 , respectively).

\section{Immunohistochemistry of Human Colorectal Tissue Using Monoclonal Anti-LPA2 Antibody}

We next examined the expression of LPA2 protein in colorectal tissues as determined by immunohisto- chemical staining, using a rat anti-LPA2 monoclonal antibody prepared against a peptide located in the C-terminal region of the human LPA2 protein. Immunohistochemical analysis in all cases revealed significant expression of LPA2 in cancer tissues as well as normal colorectal tissues (Figure 4a,b). Staining intensity was relatively strong in cancer tissues as compared with normal tissues. Moreover, LPA2 staining was mostly found in the cytoplasm in normal epithelium, whereas cell membranes showed stronger immunoreactivity than the cytoplasm in most carcinoma cells (Figure 4c). LPA2 staining was homogeneous throughout these areas and heterogeneity was not observed in any case. Adenoma tissues also showed a significant level of expression of LPA2 protein, and their staining intensity and pattern were similar to those of normal epithelium (Figure 4d).

\section{Western Blot of Human Colorectal Tissue Using Polyclonal Anti-LPA1 Antibody}

We also examined Western blot for tissue samples using polyclonal anti-LPA1 antibody. Total cell lysates from 16 individual human colorectal carcinoma tissues and the corresponding normal mucosa were analyzed for the presence of LPA1 protein. In nine of 16 cases, we found a significant decrease in the expression of LPA1 protein in cancer tissues compared to normal mucosa (Case 1, 6, 7, 12, 14, 15, 17, 18, and 21). Representative results of Western blot are shown in Figure 5 . In the remaining seven cases (Case 2, 4, 16, 20, 22, 24, and 25), the difference in LPA1 expression between normal mucosa and cancer does not seem significant, whereas there is no case that shows an increase in the expression of LPA1 in cancer tissue. Although Western blot is quantitatively less sensitive than real-time RT-PCR, these results seem to be compatible.

\section{LPA Stimulate EGFR Tyrosine Phosphorylation in Human Colorectal Cancer Cells}

LPA was previously shown to stimulate tyrosine phosphorylation of EGFR. ${ }^{26-28}$ This inter-receptor crosstalk is termed 'EGFR signal transactivation'.26-28 EGFR transactivation was linked to diverse biological process in human cancer cells, such as proliferation and antiapoptosis, and thus considered to be closely related with carcinogenesis. ${ }^{26,29,30}$ To assess the LPA receptor involvement in LPA-induced transactivation of EGFR in colorectal cancer, we investigated five human colon cancer cells, COLO320, LoVo, HT29, WiDR, and HCT116 cells. As shown in Figure 6, LPA induced significant level of tyrosine phosphorylation of EGFR in LoVo, HT29, WiDR, and HCT116 cells (all of which expressed LPA2 abundantly), whereas COLO320 cells (which expressed only little LPA2) were not transactivated 

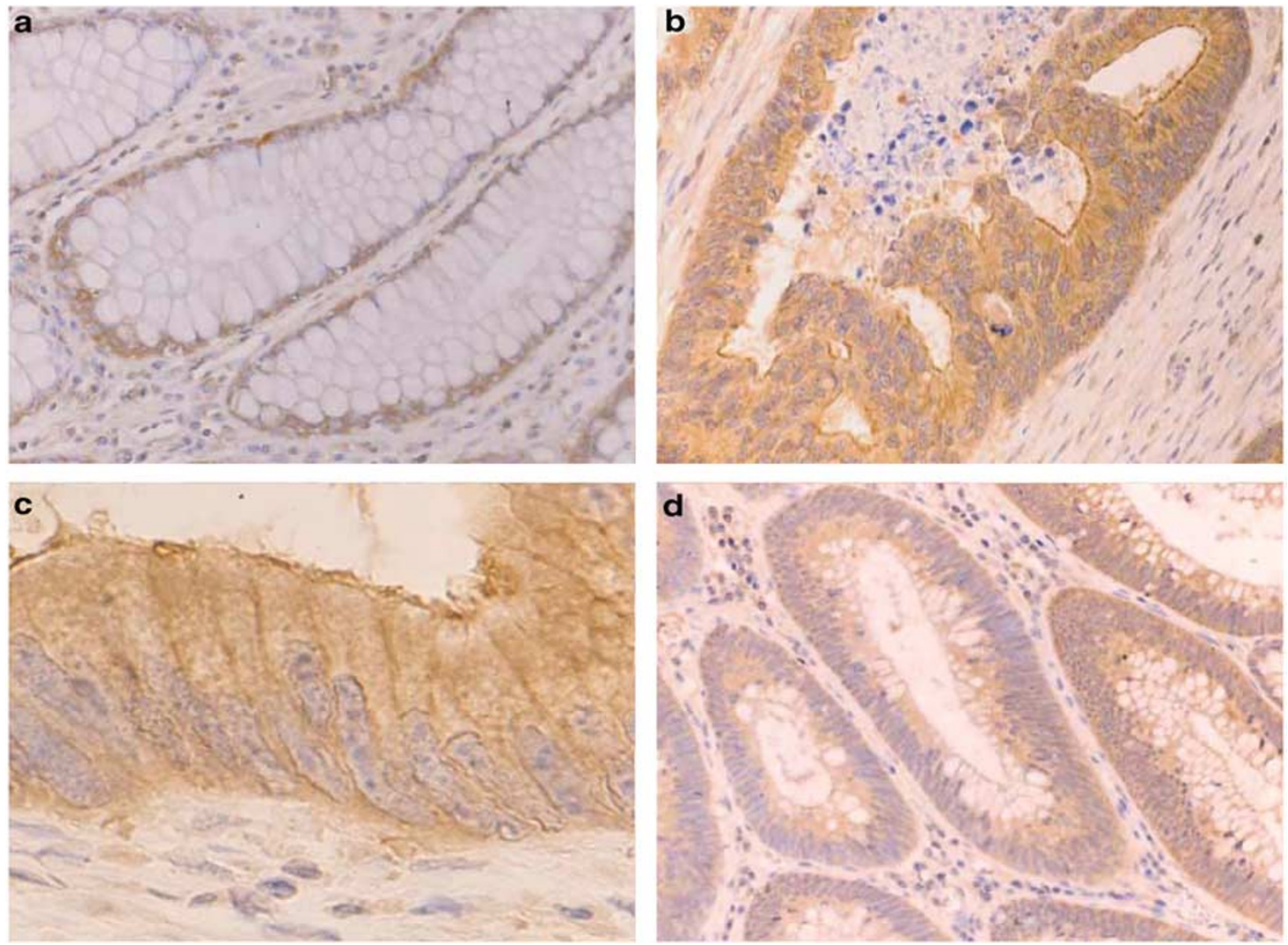

Figure 4 Immunohistochemical staining of colorectal tissues with antibody against LPA2. Colorectal cancer tissues were stained for the presence of LPA2 using a monoclonal antibody prepared against a peptide located in the C-terminal region of human LPA2. A total of 26 samples were examined and a photomicrograph of one representative case is shown: (a) normal colorectal tissue: $\times 200$ magnification; (b) colorectal cancer tissue: $\times 200$ magnification; (c) colorectal cancer tissue: $\times 400$ magnification; (d) colorectal adenoma tissue: $\times 200$ magnification.

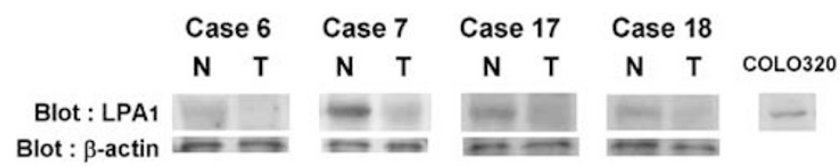

Figure 5 Western blot analysis of colorectal tissues using antibody against LPA1. Total cellular lysates from individual human colorectal carcinoma tissues and the corresponding normal mucosa were analyzed for the presence of LPA1 protein. Total protein of each sample was determined by Western analysis with antibodies to $\beta$-actin. In all, 16 matched samples were examined and four representative cases are shown. Cell lysate of COLO320 cells was used as a positive control for LPA1 protein.

by LPA. Moreover, transactivation of EGFR in HCT116 cells (that expressed all of three LPA receptors abundantly) induced by LPA was not significantly interfered with LPA1/LPA3 antagonist, DGPP. ${ }^{24}$ These data suggest that LPA2 receptor, but not LPA1 or LPA3, plays the main role in LPAinduced EGFR transactivation in colon cancer cells.

\section{Discussion}

In the present study, we used a quantitative realtime RT-PCR method to quantify, LPA1, LPA2, and
LPA3 mRNA in colorectal cancer and normal mucosa, since this assay is a rapid and sensitive method to quantify gene expression and requires smaller amounts of colorectal tissue than conventional methods. We first examined the mRNA expression in cultured colon cancer cell lines. The results obtained with real-time RT-PCR were clearly consistent with our previous data obtained by Northern blot analysis. ${ }^{22}$ We thus confirmed the reliability of our real-time RT-PCR method.

We then examined colorectal cancer tissue and normal tissue, obtained by surgical resection, by real-time RT-PCR. Our results clearly revealed that colorectal cancers showed reduced expression of LPA1 mRNA as compared with normal mucosa. Previous studies, using Northern blot analysis, have shown that, whereas LPA1 was expressed in normal colon tissue, it was expressed in only three out of nine colon cancer cell lines. ${ }^{14,22}$ These results are compatible with our present results, and strongly suggest that expression of LPA1 in colorectal tissue is reduced during malignant transformation. In contrast, colorectal cancers showed markedly increased expression of LPA2 mRNA. Using an antiLPA2 monoclonal antibody, we confirmed the high expression of LPA2 in colorectal cancer tissues at 


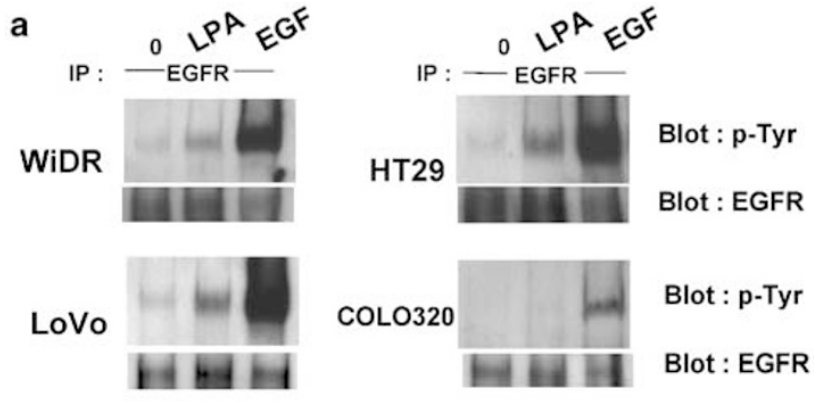

b

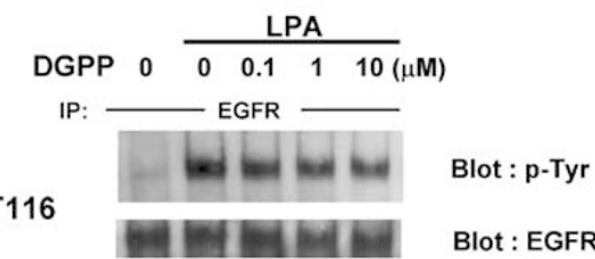

Figure 6 LPA induces tyrosine phosphorylation of EGFR in various colon cancer cells. (a) Various colon cancer cells were starved in serum-free medium overnight and treated with $10 \mu \mathrm{M}$ LPA or $10 \mathrm{ng} / \mathrm{ml}$ EGF for $2 \mathrm{~min}$. After lysis, EGFR was immunoprecipitated using polyclonal anti-EGFR antibody. Tyrosinephosphorylated EGFR was detected by immunoblotting with monoclonal antiphosphotyrosine antibody, followed by reprobing of the same filter with polyclonal anti-EGFR antibody. (b) HCT116 cells were pretreated with various concentrations of DGPP (8:0) for $30 \mathrm{~min}$ and then treated with $10 \mu \mathrm{M}$ LPA for $2 \mathrm{~min}$. After lysis, immunoprecipitation and immunoblotting were performed as described before.

the protein level. As for other tumors, whereas normal ovarian epithelial cells express LPA1 mRNA and low levels of LPA2 and LPA3 mRNA, most ovarian cancer cells express elevated levels of LPA2 and LPA3 mRNA and variable levels of LPA1 mRNA without a consistent pattern. ${ }^{6,9}$ Fang et $a l^{20}$ and Fujita et $a l^{21}$ recently reported the same findings using ovarian cancer samples and normal tissues directly obtained from patients. That is, both LPA2 and LPA3 mRNA were overexpressed in a significant portion of ovarian cancer samples compared with tissue from normal ovaries. In thyroid tissues, LPA2 mRNA expression was increased three-fold in differentiated thyroid cancer compared to normal thyroid or goiter. ${ }^{19}$ From these data, together with our results, it is thought that overexpression of LPA2 may be a common finding in a wide range of human cancer tissues and that LPA2 may be a key receptor involved in the development of various cancers. ${ }^{14,19,20}$ As for LPA3, its expression in colorectal tissues was low in both normal and cancer tissues. Although previous reports showed that ovarian cancer tissue and cell lines had high expression of LPA3, suggesting the involvement of this receptor in the carcinogenesis of ovarian cancer, LPA3 does not appear to play a significant role in colorectal carcinogenesis. ${ }^{6,9,20}$

We revealed that the ratio of LPA2/LPA1 in cancer tissue was markedly increased by as much as 18 -fold, as compared with that in normal tissue.
Since there are several reports showing opposing effects of these two LPA receptors, it may be more rational to consider the ratio of LPA2/LPA1 to understand the total effect of LPA on target cells than to consider the levels of LPA1 and LPA2 separately. It remains to be determined whether an increase in the ratio of LPA2/LPA1 contributes in any way to pathogenesis in cancer biology. One advantage of the preferential expression of LPA2 may relate to the different proliferative responses to LPA1 and LPA2. In ovarian cancer cells, the proliferative responses of high LPA1 receptor-bearing ovarian cancer cells to LPA were uniformly less than those of low LPA1 receptor-bearing lines. ${ }^{31}$ Moreover, additional transfection of LPA1 to LPA2transfected rat HTC4 cells caused a decrease in the proliferative response to LPA. ${ }^{32}$ This evidence implicates a suppressive influence of the LPA1 receptor on proliferation, compared to the LPA2 receptor. A decrease of LPA1 expression in colorectal cancer may therefore be advantageous from the standpoint of cell proliferation.

Another advantage of preferential expression of LPA2 may relate to the different protective effects against apoptosis. It has been reported that LPA has an antiapoptotic effect on various cells, although it was not satisfactorily examined which LPA receptor mediated this response. ${ }^{33,34}$ Recently, Deng et $a l^{35}$ have reported that LPA prevented apoptosis of rat intestinal epithelial cells (IEC-6) induced by radiation and chemotherapeutic agents, which was mediated through both LPA1 and LPA2. In contrast, it was reported that overexpression of LPA1 in ovarian cancer cells induced apoptosis and anoikis in an LPA-independent manner, implicating LPA1 as a negative growth receptor under certain circumstances. ${ }^{31}$ Thus, LPA1 can mediate both antiapoptotic and apoptotic effects, seemingly depending on the cell type or other circumstances. Acquiring high expression of LPA2 and reduced expression of LPA1 during malignant transformation are thought to be advantageous for colorectal cancer, as well as ovarian cancer, to survive in LPA-rich circumstances.

Similar opposing activities of the two LPA receptors were also observed in human blood T-helper cells. Freshly isolated T-helper cells predominantly expressed the LPA2 receptor, and secretion of interleukin-2 was decreased by LPA, whereas mitogen-activated T-helper cells expressed more LPA1 than LPA2, and secretion of interleukin2 was increased by LPA. ${ }^{36}$ Another report revealed that in LPA2-dominant Jurkat T cells, LPA stimulated trans-Matrigel migration and MMP functional activity, whereas in LPA1-dominant Jurkat T cells, LPA did not affect migration or MMP activity. ${ }^{37}$ These changes in the expression pattern of LPA receptors in T-helper cells in immune responses appear to be reasonable, since LPA first facilitates T-helper cell recruitment to the site of an immune response through LPA2, and then augments effector 
reactions including cytokine generation through LPA $1 .{ }^{32}$ These opposing effects of LPA transduced by LPA1 and LPA2 suggest an advantage of multiple LPA receptors specific for the same ligand in the precise regulation of complex immune responses. Such regulation may also be seen in the development and progression of cancer.

Several agonists of G protein-coupled receptors (GPCRs) were previously shown to stimulate tyrosine phosphorylation of EGFR. ${ }^{27,28} \mathrm{LPA}$ is one of GPCR agonists that induce EGFR signal transactivation. ${ }^{26-28}$ EGFR transactivation may result in raf/ras/ ERK1,2 cascade activation and lead to the increased cell proliferation and, possibly resistance to apoptosis. ${ }^{26,29,30}$ From the standpoint of LPA receptor involvement, however, LPA-induced transactivation of EGFR has not been studied yet. In our study of colon cancer cells, LPA induced transactivation of EGFR in various LPA2-expressing cells, but not in COLO320 cells that expressed only little LPA2. Moreover, LPA-induced transactivation was not interfered with LPA1/LPA3 antagonist, DGPP even at $10 \mu \mathrm{M}$. DGPP at this concentration should completely inhibit LPA3, while LPA1 may still be partly activated by $10 \mu \mathrm{M}$ of LPA, ${ }^{24}$ and thus the contribution of LPA1 could not be completely neglected. However, LPA2 receptor seems to play an important role in LPA-induced EGFR transactivation in colon cancer cells. This may be also advantageous to the preferential expression of LPA2 in colorectal cancer tissues. In addition, although LPA did not significantly transactivate EGFR in COLO320 cells, LPA induced proliferation of COLO320 cells. Therefore, both EGFR transactivation (mainly via LPA2) and direct LPA-receptor-mediated activation of Ras complexes seems to be involved in LPA mitogenic/ prosurvival effects in colon cancer cells.

It is understood that colorectal cancers develop as the result of stepwise progression through several genetic alterations. ${ }^{38,39}$ The development process involves the accumulation of genetic changes in many tumor-suppressor genes, which in turn propel the tumor down the adenoma-carcinoma sequence. Inactivation of the APC gene on chromosome 5 is widely accepted as one of the earliest steps; it can result in adenoma formation. ${ }^{40}$ Min (multiple intestinal neoplasia) mice, a murine model of human familial adenomatous polyposis, carry a dominant mutation in the homolog of the APC gene and develop multiple adenomas throughout their small and large intestine. ${ }^{41,42} \mathrm{~A}$ further interesting observation is that the number of adenomas that Min mice develop varies by as much as nine-fold depending on the genetic background of the mouse ${ }^{43-45}$ Genetic analysis has revealed that a locus on chromosome 4 designated Mom1 (Modifier of Min1) can account for $50 \%$ of the genetic variation. The secretory phospholipase A2 (sPLA2) gene has been proposed as a candidate for the MOM1 locus. ${ }^{46,47}$ Although it is unknown how this enzyme modifies tumor susceptibility, it is of interest since PLA2 is a key enzyme in the metabolism of membrane phospholipids into lysophospholipids as well as eicosanoids. This may suggest a close relation between lipid metabolism and intestinal polyp initiation and progression. ${ }^{48}$ There is a possibility that the alteration of local lipid metabolism in the colorectal mucosa, which may induce a change in the amount of LPA, and that of the expression pattern of LPA receptors may be a crucial event in the development of colorectal cancer.

In humans, the use of nonsteroidal anti-inflammatory drugs (NSAIDs) has been linked to a $40-50 \%$ reduction in relative risk for colorectal cancer. ${ }^{49}$ The molecular basis for this striking chemopreventive effect has been attributed to inhibition of cyclooxygenases (COX) and the resulting decrease in prostaglandin production. ${ }^{49}$ Consistent with this, approximately $80 \%$ of colorectal cancers have increased levels of COX-2. ${ }^{49,50}$ The critical roles of eicosanoids in colorectal tumors have therefore been much reported. However, the role of lysophospholipids, the other metabolites of phospholipids in cellular membranes, in colorectal tumors is not clear. ${ }^{51}$ The present study revealed that overexpression of LPA2 is involved in colorectal carcinogenesis, and that antagonists to the LPA2 receptor may thus be a possible candidate as chemopreventive agents against colorectal cancer. Newly discovered nonlipid agonists and antagonists for individual LPA receptors will permit more sophisticated analyses of their respective contributions to tumor biology.

\section{Acknowledgements}

This work was supported partly by a Grant-in-Aid for Scientific Research from the Ministry of Education, Culture, Sports, Science and Technology of Japan and partly by a grant from the Ministry of Health, Labour and Welfare of Japan. We thank Ms C Uchikawa and Ms K Amitani for their technical assistance.

\section{References}

1 Moolenaar WH. Lysophosphatidic acid, a multifunctional phospholipid messenger. J Biol Chem 1995; 270:12949-12952.

2 Moolenaar WH. Bioactive lysophospholipids and their $\mathrm{G}$ protein-coupled receptors. Exp Cell Res 1999;253: 230-238.

3 Goetzl EJ, An S. Diversity of cellular receptors and functions for the lysophospholipid growth factors lysophosphatidic acid and sphingosine 1-phosphate. FASEB J 1998;12:1589-1598.

4 Mills GB, Moolenaar WH. The emerging role of lysophosphatidic acid in cancer. Nat Rev Cancer 2003;3:582-591. 
$5 \mathrm{Xu}$ Y, Shen Z, Wiper DW, et al. Lysophosphatidic acid as a potential biomarker for ovarian and other gynecologic cancers. JAMA 1998;280:719-723.

6 Erickson JR, Hasegawa Y, Fang X, et al. Lysophosphatidic acid and ovarian cancer: a paradigm for tumorigenesis and patient management. Prostaglandins 2001;64:63-81.

7 Baker DL, Morrison P, Miller B, et al. Plasma lysophosphatidic acid concentration and ovarian cancer. JAMA 2002;287:3081-3082.

$8 \mathrm{Xu}$ Y, Gaudette DC, Boynton JD, et al. Characterization of an ovarian cancer activating factor in ascites from ovarian cancer patients. Clin Cancer Res 1995;1: 1223-1232.

9 Goetzl EJ, Dolezalova H, Kong Y, et al. Distinctive expression and functions of the type 4 endothelial differentiation gene-encoded $G$ protein-coupled receptor for lysophosphatidic acid in ovarian cancer. Cancer Res 1999;59:5370-5375.

$10 \mathrm{Hu}$ YL, Tee MK, Goetzl EJ, et al. Lysophosphatidic acid induction of vascular endothelial growth factor expression in human ovarian cancer cells. J Natl Cancer Inst 2001;93:762-768.

11 Fishman DA, Liu Y, Ellerbroek SM, et al. Lysophosphatidic acid promotes matrix metalloproteinase (MMP) activation and MMP-dependent invasion in ovarian cancer cells. Cancer Res 2001;61:3194-3199.

12 Schwartz BM, Hong G, Morrison BH, et al. Lysophospholipids increase interleukin-8 expression in ovarian cancer cells. Gynecol Oncol 2001;81:291-300.

13 Mills GB, Eder A, Fang X, et al. Critical role of lysophospholipids in the pathophysiology, diagnosis, and management of ovarian cancer. Cancer Treat Res 2002;107:259-283.

14 An S, Bleu T, Hallmark OG, et al. Characterization of a novel subtype of human $G$ protein-coupled receptor for lysophosphatidic acid. J Biol Chem 1998;273: 7906-7910.

15 Bandoh K, Aoki J, Hosono H, et al. Molecular cloning and characterization of a novel human G-proteincoupled receptor, EDG7, for lysophosphatidic acid. J Biol Chem 1999;274:27776-27785.

16 Contos JJ, Fukushima N, Weiner JA, et al. Requirement for the lpA1 lysophosphatidic acid receptor gene in normal suckling behavior. Proc Natl Acad Sci USA 2000;97:13384-13389.

17 Contos JJ, Ishii I, Fukushima N, et al. Characterization of lpa(2) (Edg4) and lpa(1)/lpa(2) (Edg2/Edg4) lysophosphatidic acid receptor knockout mice: signaling deficits without obvious phenotypic abnormality attributable to $\operatorname{lpa}(2)$. Mol Cell Biol 2002;22: 6921-6929.

18 Fang X, Gaudette D, Furui T, et al. Lysophospholipid growth factors in the initiation, progression, metastases, and management of ovarian cancer. Ann NY Acad Sci 2000;905:188-208.

19 Schulte KM, Beyer A, Kohrer K, et al. Lysophosphatidic acid, a novel lipid growth factor for human thyroid cells: over-expression of the high-affinity receptor edg4 in differentiated thyroid cancer. Int J Cancer 2001;92:249-256.

20 Fang X, Schummer M, Mao M, et al. Lysophosphatidic acid is a bioactive mediator in ovarian cancer. Biochim Biophys Acta 2002;1582:257-264.

21 Fujita T, Miyamoto S, Onoyama I, et al. Expression of lysophosphatidic acid receptors and vascular endothelial growth factor mediating lysophosphatidic acid in the development of human ovarian cancer. Cancer Lett 2003;192:161-169.

22 Shida D, Kitayama J, Yamaguchi H, et al. Lysophosphatidic acid (LPA) enhances the metastatic potential of human colon carcinoma DLD1 cells through LPA1. Cancer Res 2003;63:1706-1711.

23 Aoki J, Taira A, Takanezawa Y, et al. Serum lysophosphatidic acid is produced through diverse phospholipase pathways. J Biol Chem 2002;277: 48737-48744.

24 Fischer DJ, Nusser N, Virag T, et al. Short-chain phosphatidates are subtype-selective antagonists of lysophosphatidic acid receptors. Mol Pharmacol 2001; 60:776-784.

25 Chomczynski P, Sacchi N. Single-step method of RNA isolation by acid guanidinium thiocyanate-phenolchloroform extraction. Anal Biochem 1987;162: 156-159.

26 Gschwind A, Prenzel N, Ullrich A. Lysophosphatidic acid-induced squamous cell carcinoma cell proliferation and motility involves epidermal growth factor receptor signal transactivation. Cancer Res 2002; 62:6329-6336.

27 Daub H, Weiss FU, Wallasch C, et al. Role of transactivation of the EGF receptor in signalling by G-protein-coupled receptors. Nature 1996;379: 557-560.

28 Prenzel N, Zwick E, Daub H, et al. EGF receptor transactivation by G-protein-coupled receptors requires metalloproteinase cleavage of proHB-EGF. Nature 1999;402:884-888.

29 Fischer OM, Hart S, Gschwind A, et al. EGFR signal transactivation in cancer cells. Biochem Soc Trans 2003;31:1203-1208.

30 Kue PF, Taub JS, Harrington LB, et al. Lysophosphatidic acid-regulated mitogenic ERK signaling in androgen-insensitive prostate cancer PC-3 cells. Int J Cancer 2002;102:572-579.

31 Furui T, LaPushin R, Mao M, et al. Overexpression of edg-2/vzg-1 induces apoptosis and anoikis in ovarian cancer cells in a lysophosphatidic acid-independent manner. Clin Cancer Res 1999;5:4308-4318.

32 Huang MC, Graeler M, Shankar G, et al. Lysophospholipid mediators of immunity and neoplasia. Biochim Biophys Acta 2002;1582:161-167.

33 Goetzl EJ, Kong Y, Mei B. Lysophosphatidic acid and sphingosine 1-phosphate protection of $\mathrm{T}$ cells from apoptosis in association with suppression of Bax. J Immunol 1999;162:2049-2056.

34 Swarthout JT, Walling HW. Lysophosphatidic acid: receptors, signaling and survival. Cell Mol Life Sci 2000;57:1978-1985.

35 Deng W, Balazs L, Wang DA, et al. Lysophosphatidic acid protects and rescues intestinal epithelial cells from radiation- and chemotherapy-induced apoptosis. Gastroenterology 2002;123:206-216.

36 Goetzl EJ, Kong Y, Voice JK. Cutting edge: differential constitutive expression of functional receptors for lysophosphatidic acid by human blood lymphocytes. J Immunol 2000;164:4996-4999.

37 Zheng Y, Kong Y, Goetzl EJ. Lysophosphatidic acid receptor-selective effects on Jurkat $\mathrm{T}$ cell migration through a Matrigel model basement membrane. J Immunol 2001;166:2317-2322.

38 Vogelstein B, Fearon ER, Hamilton SR, et al. Genetic alterations during colorectal-tumor development. N Engl J Med 1988;319:525-532. 
39 Haydon AM, Jass JR. Emerging pathways in colorectalcancer development. Lancet Oncol 2002;3:83-88.

40 Lamlum $\mathrm{H}$, Papadopoulou A, Ilyas $\mathrm{M}$, et al. APC mutations are sufficient for the growth of early colorectal adenomas. Proc Natl Acad Sci USA 2000;97: 2225-2228.

41 Moser AR, Pitot HC, Dove WF. A dominant mutation that predisposes to multiple intestinal neoplasia in the mouse. Science 1990;247:322-324.

42 Moser AR, Dove WF, Roth KA, et al. The Min (multiple intestinal neoplasia) mutation: its effect on gut epithelial cell differentiation and interaction with a modifier system. J Cell Biol 1992;116:1517-1526.

43 Dietrich WF, Lander ES, Smith JS, et al. Genetic identification of Mom-1, a major modifier locus affecting Min-induced intestinal neoplasia in the mouse. Cell 1993;75:631-639.

44 Giardiello FM, Krush AJ, Petersen GM, et al. Phenotypic variability of familial adenomatous polyposis in 11 unrelated families with identical APC gene mutation. Gastroenterology 1994;106:1542-1547.

45 Gould KA, Dietrich WF, Borenstein N, et al. Mom1 is a semi-dominant modifier of intestinal adenoma size and multiplicity in Min/+ mice. Genetics 1996; 144:1769-1776.

46 Gould KA, Luongo C, Moser AR, et al. Genetic evaluation of candidate genes for the Mom1 modifier of intestinal neoplasia in mice. Genetics 1996; 144:1777-1785.

47 MacPhee M, Chepenik KP, Liddell RA, et al. The secretory phospholipase A2 gene is a candidate for the Mom1 locus, a major modifier of ApcMin-induced intestinal neoplasia. Cell 1995;81:957-966.

48 Watson AJ, Dubois RN. Lipid metabolism and APC: implications for colorectal cancer prevention. Lancet 1997;349:444-445.

49 DuBois RN, Giardiello FM, Smalley WE. Nonsteroidal anti-inflammatory drugs, eicosanoids, and colorectal cancer prevention. Gastroenterol Clin North Am 1996;25:773-791.

50 Eberhart CE, Coffey RJ, Radhika A, et al. Up-regulation of cyclooxygenase 2 gene expression in human colorectal adenomas and adenocarcinomas. Gastroenterology 1994;107:1183-1188.

51 Hla T, Lee MJ, Ancellin N, et al. Lysophospholipidsreceptor revelations. Science 2001;294:1875-1878. 